\title{
A Common Awareness and Knowledge Platform for Studying and Enabling Independent Living - CAPSIL
}

\author{
Bennis C, McGrath D, Caulfield B, \\ School of Public Health, Physiotherapy \& \\ Population Science \\ UCD, Dublin, Ireland. \\ caoimhebennis@gmail.com, denisemcgrath@hotmail.com \\ b.caulfield@ucd.ie,
}

Abstract:

The population of the world is growing older, and the balance of old to young is shifting so that by 2050 over $30 \%$ of the population is expected to be over 60 years old[1], with particularly high ratios of old to young in the EU, USA and Japan. CAPSIL is an FP7 Coordinating Support Action that incorporates a strategic international coalition of University and Industrial partners that already have extensive teams developing hardware/software/knowledge solutions to independent living based on user requirements. CAPSIL has two fundamental goals:

1. To carry out an analysis of the state of the art with regards to technology, healthcare and public policy in the EU, US and Japan for enabling independent living for older adults. Based on this analysis, develop a detailed roadmap for EU research to achieve effective and sustainable solutions for independent living

2. To support aging research by proposing procedures to incorporate all of these diverse solutions into WiKi entries (CAPSIL WiKi). It is our hope that these CAPSIL WiKi's will enable researchers and the ICT industry to get the information they need to quickly and easily test solutions for prolonging independent living within the many and various heterogeneous communities.

In this paper we will summarise the principal findings of the CAPSIL Roadmap and present an overview of the main research gaps and recommendations for policy and research development. Finally, we will introduce the CAPSIL WiKi infrastructure.

Keywords- ageing, healthcare, ICT, independent living, monitoring systems, Pervasive Health, Policy, Software, Telemedicine, Wiki, Wireless Networks

\author{
Knapp B, Coghlan N \\ School of Music and Sonic Arts, SARC, \\ QUB, Belfast, \\ Northern Ireland. \\ b.knapp@QUB.AC.UK, ncoghlan02@QUB.AC.UK
}

\section{INTRODUCTION}

As the world's population ages, with particularly high ratios of old to young expected in the EU, USA and Japan [2], measures need to be taken to ensure that the older population are enabled to live longer independently in a safe, proactive and healthy environment, while decreasing the cost burden on public health systems [3]. Eric Dishman, is an Intel Fellow and director of Health Innovation and Policy for Intel's Digital Health Group, who developed a graph (Fig.1) representing quality of life levels versus daily cost for patients in acute, residential and home care. The result shows highest quality of life with lowest cost factor for patients in the home care setting. The graph shows highest quality of life with lowest cost factor for patients in the home care setting. This concept underpins the initiation of EU strategies such as i2010 and FP7 to include research and development $(\mathrm{R} \& \mathrm{D})$ projects focused on finding solutions to enabling older people to live longer independently at home. The findings of the CAPSIL roadmap identifies the existing obstacles to the attainment of this goal internationally and within the EU, and makes recommendations to overcome them.

CAPSIL is an FP7 Coordinating Support Action within the

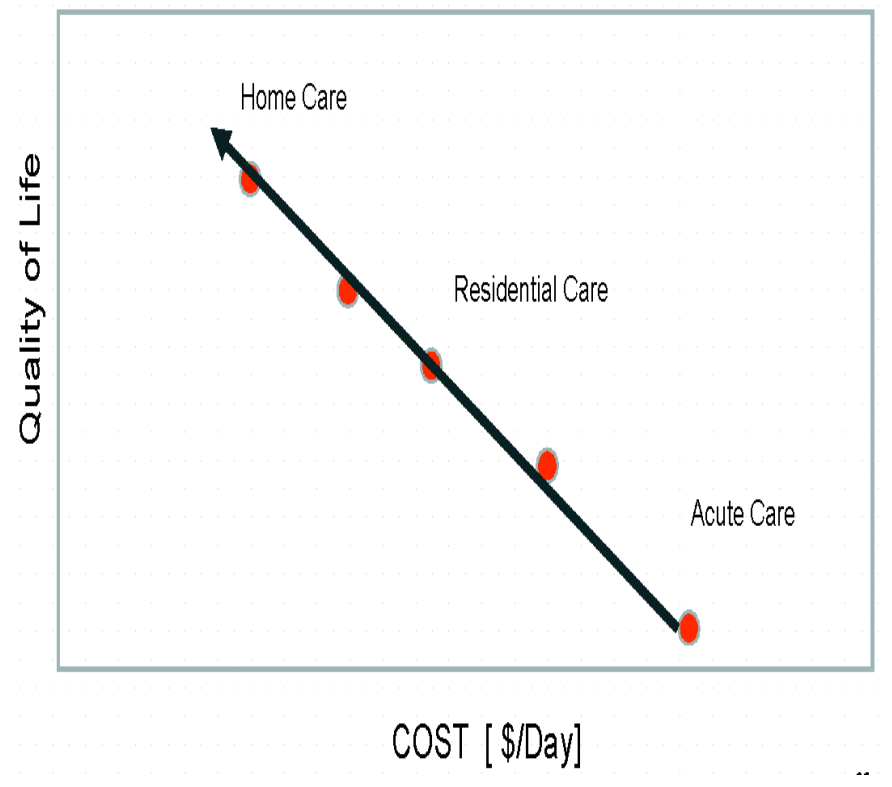

Figure 1. Impact Assessment $₫$ Eric Dishman 
specific programme of "cooperation" and research theme "ICT". The CAPSIL consortium is made up of University and Industrial partners actively involved in Ageing related Information and Communication Technology (ICT). CAPSIL aims to promote awareness and knowledge of the current activities in Ageing related ICT, while conducting analysis on the current barriers to development within the EU, US and Japan. The EU, US and Japan are leading the way in research and policy internationally. By conducting our analysis across all three regions we hope to build an international knowledge platform which will increase awareness and promote international collaboration on $\mathrm{R} \& \mathrm{D}$ projects. The aim of this paper is to introduce a vision of proactive, technology-based, healthcare monitoring systems within the home in the year 2025. We will then present the current gaps in technology, policies and the market, identified by the CAPSIL consortium, and make a series of recommendations for actions that are necessary to enable the progress of $R \& D$.

An essential element of CAPSIL'S work is the development of a WiKi (www.capsil.org/capsilwiki), an online resource corresponding to the CAPSIL roadmap document. The information on this $\mathrm{WiKi}$ will initially represent the current activity within ageing related ICT. However, it is our intention that those involved in this area will update the $\mathrm{WiKi}$ as technology develops, maintaining the relevance of the information on R\&D and issues in this area. The WiKi will be discussed further towards the end of the paper.

\section{METHODOLOGY}

The information presented in this paper is a summary of the findings of the original CAPSIL Roadmap. The roadmap was originally broken down into separate chapters: Background \& Problem Definition, Intervention, Visioning, Monitoring Systems, Wireless Sensor Networks, Software and Strategy. Each Chapter included research into Socioeconomics, Technology and Policy relating to each topic. One member of the CAPSIL consortium from the EU, US or Japan initially compiled each chapter. This first draft was then reviewed twice more by different members of the consortium ensuring that it was reviewed at least once from EU, US and Japanese perspectives. Research was conducted through a review of the current available literature and web resources. While further information on ongoing R\&D was compiled from attending international workshops, visiting centre's for ageing R\&D as well as activity within CAPSIL member's individual research centres.

This information was then input into the CAPSIL WiKi and supplemented with additional information surrounding particular topics introduced within the Roadmap. This extra information stems from the initial review of literature and web resources conducted for the Roadmap, which was deemed unnecessary for inclusion in the Roadmap dialogue but of interest and benefit as further background information for the reader.

\section{VISIONING}

Preventative health models are a focus of EU strategy particularly since 2005 with the launch of i2010: Independent living for the Ageing society. CAPSIL envisaged a number of scenarios describing a day in the life of a group of elderly men and women, of various backgrounds, living with monitoring systems and technology interventions at home and on the move. These visions are an introduction to how technology could help, in the foreseeable future, to maintain independent, high-quality life for elders. They are based on a number of key themes identified as important aspects for high QOL. such as: Meaning and Identity in late life, Memory and Remembering, Dignity and privacy, Independance and Social Interaction, Activity (Active Lives vs Succumbing to old age) and the importance of Family relationships. Here we will introduce an excerpt from the day in the life of Tom Capsil, a 76-year-old American man, who suffers from a congestive heart condition that was contributed to by his obesity (see Table 1). Some of the technologies incorporated in these scenarios are already deployed in the field or in the laboratory, several are part of focus-advanced research and others need to be investigated. The described environment is focused on unobtrusive sensing and assessment and on minimally obtrusive interventions.

The reader may notice the unobtrusive and subtle way that the system adapts to the elder's values and preferences. The economic feasibility of the technology-based approach and clinical effectiveness require further research. There are many gaps between today's technology and those represented in Tom's world:

- Auto-pilots for motor vehicles are not commercially available.

- Attention-span devices (for cars for example) are in development but are not commercially available.

- The personal assistant Carobot. Personal robots are not available for consumers. TWENDY-ONE[4], just one of the robots currently being developed in Japan, provides strength for assisting with transfers and dexterity to assist with feeding.

- Many of the unobtrusive physiological and biomechanical monitoring systems mentioned in this scenario (eg blood pressure monitor in watch strap, urinalysis equipped toilet) are not in a mature stage of development.

- Physiological measurements are not currently used as input to coaching or cognitive monitoring devices. The two types of devices operate seperately and need to be brought together.

- Integrated tele-health monitoring and care delivery systems have been demonstrated yet are not widely available on a commercial basis.

- Medication Reminders are available internationally commonly using watch reminders or SMS reminders, however intelligent integrated systems such as described in Tom's story are not available.

\section{MAIN PROBLEMS DEFINED}

Interventions for home health typically rely on monitoring data from the home as inputs for dynamic alerts, treatment 
TABLE I. AN EXCERPT FROM TOM'S STORY.

On his way home from his Thursday consulting session, Tom Capsil turned off the autopilot in his leased electric carenjoying the feeling of control. His coach encourages him to practice as much as possible without automation, to maintain his driving skills. Tom allows himself the luxury of the leased car since his 75 th birthday while he is still commuting to his part-time work - helping a couple of young kids starting their own bakery... a chime suddenly interrupted Tom's thoughts - the Automobile Safety System detected an attention lapse by his eye movement pattern and by EEG measurements using remote laser sensors. As he pulls up to the house both the garage door and front door open as soon as the security system in his house detects the RFID signal transmitted by his watch. Tom, supported by a smart cane, walks into the kitchen later than usual and was greeted by the friendly voice of "Carobot", his robotic valet, gently reminding him of the session with his remote coach. But Tom did not start his coaching session yet - he was a little embarrassed since he did not yet do his daily exercise. Instead, using a voice command, he started his exercise game routine, as this game-based system was actually fun! It was physically and mentally challenging, without embarrassment, within the privacy of his bedroom. He was amazed because he was clearly improving - imagine at his age! Today, he pushed himself particularly hard because he wanted to surpass his previous record. He can push himself hard because Tom is well aware that the system monitors his vital signs and does not let him overdo it. This close monitoring is particularly important because of his congestive heart condition diagnosed a couple of years ago.

The results of his exercise were instantly communicated to his coach, and when Tom actually initiated the session there was already a message praising him for his accomplishments. The coaching system had already incorporated today's weight measurements (automatically assessed by the load cells in the bed as well as a scale in the floor mat in the bathroom), blood pressure - measured by a sensor in his watchstrap, and chemical analysis for sodium ion concentration in his urine performed by the toilet. The coaching system, as well as his coach, was pleased with his outside activities, socialisation and diet. Even his balance had improved so much that his Carobot, a recent robotic addition to his house, just stands by when he gets up at night to go to the bathroom, rather than providing him with mobility support.

Tom is a different man now, with a new outlook on life. His activities are continuously monitored, including his nutrition and bathing. The system has adapted to his habits and only responds when something out of the ordinary happens. Even then, before an alert is sent out, Tom is able to cancel it if he is OK. Tom feels much safer than before and has become much more active, although he allows only a small number of people to have access to this information. In a funny way, this monitoring system brought him close to his son. Tom learned very quickly that Michael actually checks his status several times a day, even when he doesn't call. The thought brings tears to Tom's eyes. Approaching the door with his coat on, the context-aware reminder system sounds a gentle alert reminding him to take his medication. Even though it is 30 minutes before the medication is due, the system figured out that Tom is likely to leave his house without taking his medication on time.

recommendations and advice. They involve the active participation of the patient and typically support the individual in adhering to health behaviour goals. Focus group studies have shown that users primary concerns were QOL and maintaining independence, while disease management was a focus for carers [3]. Home health interventions need to maintain a balance of elders and carers goals and may include aspects of safety interventions such as fall detection/ prevention and wander detection alerts, disease management systems for chronic conditions and wellness management systems addressing weight, physical and coginitive exercise. The CAPSIL roadmap has addressed different technological aspects necessary to implement these interventions. These are Monitoring Systems, Wireless Sensor Networks and Software and Interface development. There were a number of common barriers identified in the development and implementation of these aspects of the interventions described above.

\section{A. Developing User Based Interventions -}

The development of effective interventions that promote health and independence in older adults, based on a detailed analysis of patient's needs particularly from the user's perspective. Focus groups and feedback from participants in pilot activities are necessary to direct this development. To date, inadequate comprehension of user needs and poor demands for products and services to enable independent living are partly explained by the fact that the industry tends to be dominated by suppliers providing a technology-push, rather than a demand-pull approach, causing user disappointment [5].

\section{B. Usability -}

Home intervention technology requires the active participation of the elderly person or carer, which requires a level of capability and confidence with the use of the technology. These issues need to be addressed from both perspectives. Developers need to create an accessible interface for the target user which can address varying levels of sensing and physical disability while easy to use. In parallel, increasing awareness and education in the use of technology needs to be addressed at user and community level. In a recent study by Steele at el, 2009 [6], where the elderly person's perception and acceptance of using wireless sensor networks to assist healthcare were investigated, it was suggested by the participants that simplicity in the design would have a positive effect on the system's ease of use. Whilst several participants questioned their own ability to successfully interact with the technology in question, they also expressed a willingness to be trained.

\section{Pilots and Trials -}

It is generally accepted that trials and pilots have happened in a fragmented fashion with a lack of coordination between the EU, US and Japan. In a recent report by Shekelle P.G et al 2006 [7], where costs and benefits of health information 
technology (HIT) in a variety of healthcare settings was examined through a review of 256 studies. No studies were identified "outside of those from a handful of HIT leaders" that would allow a reader to make a determination about the generalisable knowledge of the study's reported benefit. There have been an insufficient number of studies, which show definitive return of investment, while reporting of results from research projects such as EU projects is poor and needs to be improved. Tools such as the CAPSIL WiKi and websites such as www.ict-ageing.eu, which document current activity in a modifiable format, need to be promoted, encouraging viewers to update the site with new research findings and initiatives.

\section{Interoperability and Integration -}

Intervention systems need to be integrated into Personal Health Records (PHR's) that are linked to clinicians' electronic medical records (EMR's). This requires the standardisation of terminology and data definitions and cross-compatibility of health information systems. The lack of standard metrics for quality of service assessment impedes the evaluation and obscures the progress of technology adoption and utility [8]. In addition, the systems need to be modular and yet holistic. The majority of older adults have at least 2 chronic conditions and intervention systems should coordinate care across diseases.

\section{E. Business Models / Reimbursement-}

It has generally been difficult for disease management systems to be profitable as a business. So far cost savings have been shown in terms of reduced hospital admissions and length of stay on pilot activity in the UK. However, cost savings are difficult to show in the evaluation of any computer system. Frequently, those purchasing a system may not be the ones who benefit from it. Also, unless exiting workflows are adapted to reap the benefits of the new approach, it is unlikely that there will be cost benefits. Physicians perceive a lack of financial support and high investment costs required for implementing electronic medical records (EMR), e-prescribing and decision support tools, on top of the additional costs that are required to maintain the systems [9].

\section{F. Privacy and Security -}

For intervention systems to be effective they must be tailored to individuals needs. However issues of what to share and with whom, how appropriate this sharing is, and also the levels of security involved are in themselves quite complex matters. It is imperative that elderly persons feel safe and assured within monitored environments as mistrust can lead to withholding of information, disclosure of misleading information to healthcare providers, or avoidance of the healthcare system entirely [10] More work must be done to determine optimal default privacy models that both protect and also best meet the data sharing needs for optimal care. We primarily need advances in easy and accurate authentication, user modelling that incorporates user preferences for data sharing, as well as legal privacy protections and standards.

\section{G. Legislative issues -}

A full legal framework for aging-related ICT is required for the vision of independent living to be realised. Regarding cross-jurisdictional healthcare, privacy and accountability i.e. scenarios are possible in which an individual might be receiving health advice from a healthcare professional outside their country of residence (via a Telecare system) based on information provided by a $3^{\text {rd }}$ party monitoring system.

\section{GAP ANALYSIS RELATING TO SPECIFIC DEVELOPMENT AREAS}

The CAPSIL roadmap concentrated on a number of key areas; Interventions, Monitoring Systems, Wireless Sensor Networks and Software and Interfaces, which contribute to the provision of an integrated home monitoring system. Collectively these areas are hindered by the barriers and gaps presented in the previous section. However, this section details the main gaps and recommendations specific to each area:

\section{A. Intervention Systems}

- Development of multi-pronged systems capable of handling a selection of conditions and tailored to the individual.

- $\quad$ Pilot studies showing effectiveness of interventions e.g. pervasive monitoring of $\mathrm{CHF}$, leading to large clinical trials.

\section{B. Monitoring Systems / Wireless Sensor Networks}

- $\quad$ Autonomic networks - necessary to allow easy scaling and maintenance of large scale deployments. These networks should be able to self-monitor, analyse the input data, plan based on this analysis and then execute this plan.

- $\quad$ Power and Battery optimisation - again necessary for long-term, low maintenance deployments. Current areas of research include: power scavenging, photovoltaic cells, temperature gradient, wind/air flow, vibration, micro scale batteries, micro-fuel cells, electromagnetic power distribution.

- Biocompatibility- long term pervasive monitoring of humans may require sensors to be in body contact for extended periods of time. This could lead to problems such as itching or rash (due to sensor/skin contact) with further complications if implantable sensors are to be considered. Also long-term effects of Radio Frequency (RF) signals on the human body.

- Software development environments to allow optimisation of large scale sensor deployments in potentially adverse conditions.

- Improving of end-to-end reliability and robustness. As the size of sensor networks increases (allowing for more detailed monitoring) so the networks data overhead also increases. These issues may be addressed through dynamic routing and power management, with electromagnetic immunity also of importance.

- Information Fusion - sensor networks may consist of a variety of 'weak' sensors whose output is combined to give a 'strong' picture of overall activity. There is 
already an extensive body of research into information fusion but this must be applied to independent living systems. At the other end of the information fusion tunnel is the fusion of 'presentation level' information which combines these multiple sensor outputs into meaningful, context relative visualisation of information for the end user, in order to facilitate effective decision making.

- Multi-person tracking - a technically challenging problem is economically feasible, unobtrusive monitoring of multiple individuals living in the same dwelling.

- Development of Body Sensor Networks - ideally sensor devices should be pervasive and unobtrusive, such as woven into garments or embedded in everyday objects. Much work is needed in order to bring devices of this nature to market.

\section{Software and Interfaces}

- More work on mobile/outdoor capable sensing systems - current systems mainly location based, there is a need for smooth transition between mobile and static sensing systems.

- User centric design - although mentioned already it is of particular importance in this area.

- Round trip clinical validation of data inference.

- Effective techniques to manage and retrieve information from huge archives of data, potentially in distributed databases.

- Research into Artificial Intelligence systems for user interaction/context awareness, robotic control and
Kansei-based interfaces.

- Research into robots for assisted living - physical support, monitoring, interaction.

\section{OBJECTIVES OF EU ACTION ON COORDINATING RESEARCH (EU, US, JAPAN)}

An important focus in the development of the CAPSIL Roadmap was attending workshops, promoting international awareness of developments within research into ICT enabled independent living, as well as to help policy makers in the EU, US and Japan coordinate research and funding agendas. Based on the analysis of the current international situation surrounding ICT and ageing the CAPSIL consortium have compiled a list of objectives for EU policy as follows:

\section{A. Main policy objectives}

- To enhance cooperation between the EU, US and Japan with a view to avoiding duplication of effort resulting in financial, time and labour savings.

- High level cooperation will be required between policymakers in the EU, US and Japan in order to implement international standardisation and interoperability legislation as well as legislation addressing inter-jurisdictional healthcare and telehealth.

- To facilitate and promote development and deployment of ICT for independent living.

- To promote standardisation and interoperability of independent living and healthcare information systems and technologies.

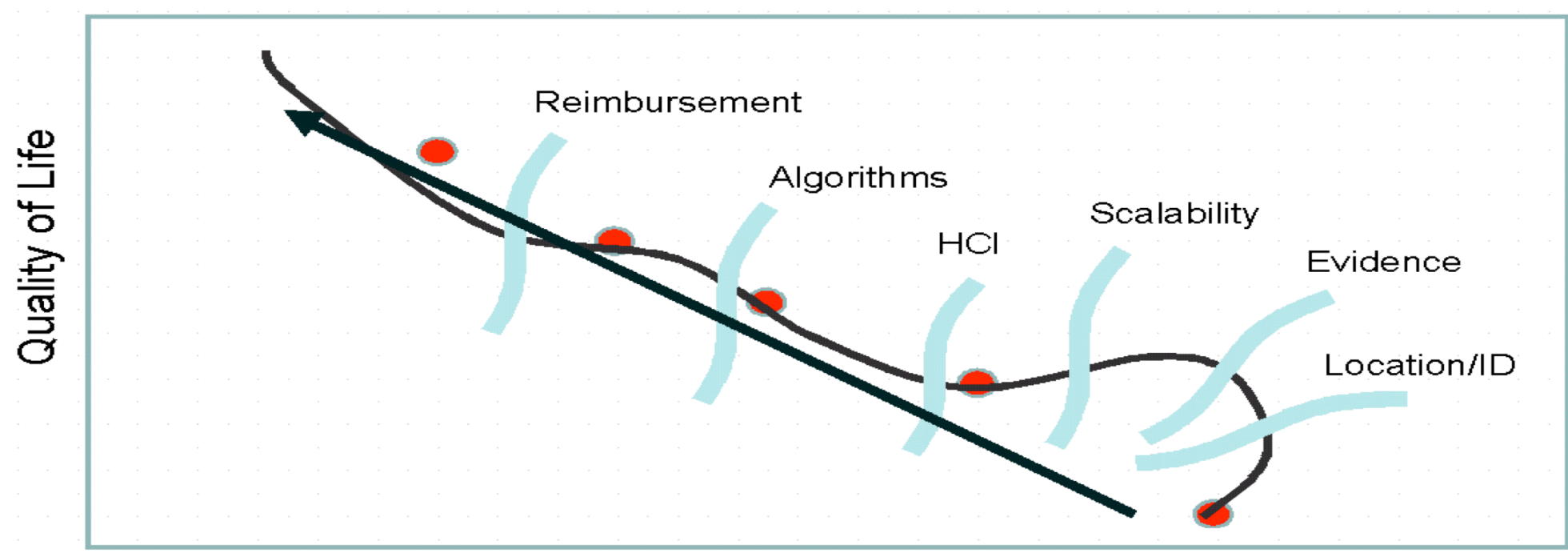

\section{COST [ \$/Day]}

Figure 2. Impact assessment graph CAPSIL Roadmap (C) Misha Pavel

Note: L1 $=$ straight line as represented in Eric Dishman's Graph $\mathrm{L} 2=$ Curved line representing various obstacles and cost involved in achieving low cost high quality living within the home setting. 
- To gather large-scale data on effectiveness of ICT for independent living.

- The Standardisation and promotion of computerised Electronic/Personal Health Records (EHR, PHR) would promote interoperability between healthcare organisations and allow leverage of data by home healthcare and Ambient Assisted Living systems. Currently there is no legislation within the EU to promote the use of these systems and the major implementations are from commercial companies: Google Health ${ }^{1}$, Microsoft HealthVault ${ }^{2}$.

- Increased funding and promotion of preventative healthcare strategies and intervention systems to manage chronic conditions.

- The development of legislation and international agreements dealing with trans-jurisdictional healthcare, telecare and ehealth. This needs to deal with accountability, reimbursement and insurance.

\section{INITIAL ASSESSMENT OF IMPACTS}

The main practical impact of no action would be to leave the current fragmented situation in place, with the corresponding impacts of inefficient use of resources and potential collapse of healthcare systems due to overburdening. In a European overview report by Steg [11], it is predicted that the EU will face a rise of $2.2 \%$ of GDP per capita up to the year 2050 , starting from $6.6 \%$ in 2000 . The rise of several countries within the EU will range from 1.7 to 3.9 percentage points of GDP.

In order to tackle these issues the EU has introduced a series of initiatives such as new EU initiative The Ambient Assisted Living (AAL) Joint Programme ${ }^{3}$ and FP7 funded research activity for health inclusion, embedded systems and micro-nano-activity. AAL is a Member State driven R\&D funding programme that is hoped to complement the longer term research on ICT and ageing within the FP7 initiative. Another initiative is the ICT Policy Support Programme (ICT PSP) who define their purpose as "stimulating innovation and competitiveness through the wider uptake and best use of ICT by citizens, governments and businesses"[12].

An impact on the formulation of European policy in this area is the goal of the new EU Health Strategy [13], that EU action should focus primarily on the causes of ill-health, rather than attempting to have disease-specific strategies at EU level. This new strategy intends to supporting healthy ageing by "promoting health throughout the lifespan, aiming to prevent health problems and disabilities from an early age, and tackling inequities in health linked to social, economic and environmental factors".

Based on the analysis of the current gaps in technology, R\&D and Policy, CAPSIL has produced a modified graph (fig 2) of Eric Dishman's idea of changing health care effects on

\footnotetext{
${ }^{1}$ https://www.google.com/health ${ }^{2}$ www.healthvault.com

3 http://www.aal-europe.eu/
}

cost and the individual's QOL (fig 1). This modified graph represents a more realistic vision of the path to fully incorporated technology-assisted independent living. Moving elderly patients from acute care, to residential care, to living at home independently will not produce a linear decline in cost, as represented by L1 in the graph. Rather, there will be periods of surging costs as barriers are encountered, followed by periods of cost saving. This is portrayed by the undulating quality of L2. Examples of such barriers are included on the graph. This is an important consideration for policy makers and market stakeholders, as the initial high cost factor required will enable the development of solutions to lower long term costs.

\section{CAPSIL WIKI}

Although vast quantities of money is clearly being invested in ICT for the elderly world-wide, the over-all information that can be gleaned from these projects to assess true advancement in the area is fragmented at best. A system that emphasises competition rather than collaboration leads to duplication of research efforts and precludes an expedited path toward the discovery of solutions to important research questions that could change the ageing landscape [14]. World-wide research collaboration and technological progress can be facilitated by promoting awareness among a broad audience to include health professionals, academic researchers and industry partners regarding pre-existing data that cross disciplinary boundaries. Information on current or future home health solutions for the elderly also needs to be available to the elderly themselves, and to their carers which would serve to educate and empower both parties. . The robustness of aging-related ICT research has, up to now, been compromised by the lack of data analysing patient's perceptions of this mode of healthcare[15]. A freely accessible, inclusive resource where they can assimilate the current state of the art for themselves, and possibly even contribute, could reconcile this problem. The CAPSIL wiki was developed to this end.

A wiki is a web-based, open source knowledge management tool that allows for collaborative authoring environments[16]. This tool requires no detailed knowledge of complex web programming techniques for users who wish to post information to the site. It is easy-to-edit and track changes made by visitors to the site. The literature contains several examples where wikis have been used to promote effective knowledge management and efficient systems in healthcare settings[16-19]. Of particular note is the study carried out by Carvalho et al, 2010[14] who used a wiki-based platform to establish a collaborative environment for the sharing of information on epidemiological and clinical research data sets, with notable success.

The use of the Internet as a platform for presenting ongoing research initiatives is an increasingly common method for research dissemination. The ICT \& Ageing - European Study on Users, Markets and Technologies [20] is a project funded within the i2010 initiative which has compiled an online database of current R\&D within the 14 EU member states, the US and Japan. This website encourages visitors to comment or provide further information on research initiatives which has 
the advantage of providing an ongoing review of the information they plan to include in their final study.

The development of the CAPSIL WiKi is an integral part to the application of the CAPSIL Roadmap. The content of the WiKi is currently based on the information provided within the Roadmap. The main topic areas included in the WiKi are: Aspects of Ageing, Interventions, Enabling Technologies, Ageing Scenarios and International Pilot Activity. Where it was not possible to include every detail or further discourse on a topic within the roadmap, there is extra information available on the WiKi. The availability of this information is signposted within the document and there are links embedded in the text for easy navigation. Therefore, it provides an easily accessible, comprehensive database of the state of the art technology as well as background information on the demographic, social, health and policy issues associated with ICT and Ageing, accessible by potential stakeholders of all backgrounds. In this way the CAPSIL WiKi offers a more comprehensive knowledge platform with links to individual studies such as the ICT and Ageing study[20] mentioned above.

The CAPSIL roadmap presents a series of recommendations based on the current situation in 2010. However, as the level of activity is increasing within ageing related ICT, stationery roadmap documents such as CAPSIL or AALIANCE may quickly become redundant as events and developments shift the focus of R\&D. By developing an online knowledge platform in the format of a WiKi, which is based on the concept of easy access and editing, it is possible for CAPSIL to continue to be relevant in parallel with research advances. As the CAPSIL WiKi is specific to topics related to ICT and ageing, the CAPSIL moderator reserves the right to screen and edit content, which is not suitable. We invite readers to visit the CAPSIL WiKi at: www.capsil.org/capsilwiki to contribute their knowledge by adding or editing content. There is a getting started section which will assist users in uploading content which can be accessed from the main page.

\section{CONCLUSIONS}

"CAPSIL" stands for the International Support of a Common Awareness and Knowledge Platform for Studying and Enabling Technology. In this paper we have presented a summary of the findings and recommendations put forward by international members of the CAPSIL consortium. The focus and drive towards developing ICT based strategies to enable elderly people to live longer, happier, independent lives at home, is getting stronger. However, the effort to create new solutions tends to be fragmented and researchers face numerous barriers within the social, technological and public policy domains. In order to overcome these issues in relation to ageing research, we must work together at EU and international levels to develop and implement social, health, technology and policy reforms. CAPSIL have taken steps to create an awareness of activity and issues surrounding ICT and Ageing, through the development of a CAPSIL WiKi. We ask readers to take the next step and consolidate the information presented on the WiKi with further knowledge and information to maintain a relevant and useful resource.

\section{REFERENCES}

1.World Population Ageing: 1950-2050. United Nations Economic \& Social Affairs: Population Division 2002; Available from: http://www.un.org/esa/population/publications/worldageing19502050/ [accessed 02/02/2010].

2.United Nations, World Population Prospects: 2008 Revision. 2009; Available from: http://www.un.org/esa/population/unpop.htm [Accessed 25/02/10].

3. Steg, H., et al., Europe is facing a demographic challenge Ambient Assisted Living offers solutions. IST Project Report on Ambient Assisted Living March 2006.

4. Twendy-one. WASEDA University Sugano Laboratory TWENDY team; Available from: http://www.twendyone.com/concept e.html [accessed 28/2/2010].

5. Chan, M., et al., Smart homes - Current features and future perspectives. Maturitas, 2009. 64(2): p. 90-97.

6. Steele, R., et al., Elderly person's perception and acceptance of using wireless sensor networks to assist healthcare. International Journal of Medical Informatics, 2009. 78: p. 788-801.

7. Shekelle, P., S. Morton, and E. Keeler, Costs and benefits of health information technology. Evidence Report/Technology Assessment, 2006(132): p. 1.

8. Ackerman, M., et al., Developing Next-Generation Telehealth Tools and Technologies: Patients, Systems, and Data Perspectives. Telemedicine and e-Health, 2010. 16(1).

9. Anderson, J. and E. Balas, Computerization of Primary Care in the United States. International Journal of Healthcare Information Systems and Informatics, 2006. 1(3): p. 1-23.

10. Courtney, K., Privacy and senior willingness to adopt smart home information technology in residential care facilities. Methods of Information in Medicine, 2008. 47(1): p. 76-81.

11. Steg, H., et al., Europe is facing a demographic challenge Ambient Assisted Living offers solutions. IST Project Report on Ambient Assisted Living 2006.

12. ICT Policy Support Programme. EU Initiative; Available from: http://ec.europa.eu/information society/activities/ict psp/about/index en.htm [accessed: 26/02/2010].

13. EU Health Strategy - Together for Health: A Strategic Approach for the EU 2008-2013. 2007; Available from: http://ec.europa.eu/health/strategy/policy/index en.htm [accessed 26/02/2010].

14. Carvalho, E.C.A.d., et al., Application Description and Policy Model in Collaborative Environment for Sharing of Information on Epidemiological and Clinical Research Data Sets. PLoS ONE, 2010. 5(2): p. e9314.

15. Williams, T., C. May, and A. Esmail, Limitations of patient satisfaction studies in telehealthcare: a systematic review of the literature. Telemedicine Journal and e-Health, 2001. 7(4): p. 293-316.

16. K. Watson and C. Harper, Supporting Knowledge Creation: Using Wikis for Group Collaboration Research Bulletin, 2008(3).

17. Lau, A., Implementation of an onto-wiki toolkit using web services to improve the efficiency and effectiveness of medical ontology co-authoring and analysis. Informatics for Health and Social Care, 2009. 34(1): p. 73-80.

18. Maier, H., et al., LitMiner and WikiGene: identifying problemrelated key players of gene regulation using publication abstracts. Nucleic acids research, 2005. 33(Web Server Issue): p. W779.

19. Meenan, C., et al., Use of a Wiki as a Radiology Departmental Knowledge Management System. Journal of Digital Imaging: p. 1-10.

20. ICT \& Ageing - European Study on Users, Markets and Technologies: Preliminary Findings. 2008; Available from: http://www.ictageing.eu/?page id=246 [accessed 26/02/10]. 